immer näher dem Horizonte, und theils durch die Morgendämmerung, theils durch Mondschein beeinträchtigt, stattfinden inussten, konnte der Schweif oft nur geahnt werden. Der Comet besass einen ziemlich sicher zu beobachtenden Kern 6.-7. Grösse, so dass die Beobachtungen trotz der Nähe des Horizonts recht gut ausfielen. Nur wurde die Zahl der passenden Anschlusssterne dadurch sehr verringert,

Königsberg 1895 Dec. 8. so dass der Comet Nov. $3^{\circ}$ an einen beinahe $13^{\text {m }}$ abstehenden Stern ringmikrometrisch angeschlossen werden musste. Mehrere Beobachtungen an anderen Tagen wurden bald nach Beginn durch eintretende Bewölkung vereitelt. Die ietzte Beobachtung stimmt fast genau mit der Ephemeride von Prof. I.amp in A. N. 3320 uberein.

\title{
Osservazioni della Cometa 1895 Il (Swift)
}

fatte in Teramo al micrometro filare del 15.5 inch di Cooke.

Latitudine $\varphi=+42^{\circ} 39^{\prime} 27^{\prime \prime}$. Riduzione dei tempi a Berlino $=-1^{\mathrm{m}} 2 \mathrm{1}^{\mathrm{s}}$.

(Continuazione, vedi A. N. $33 \times 3$ ).

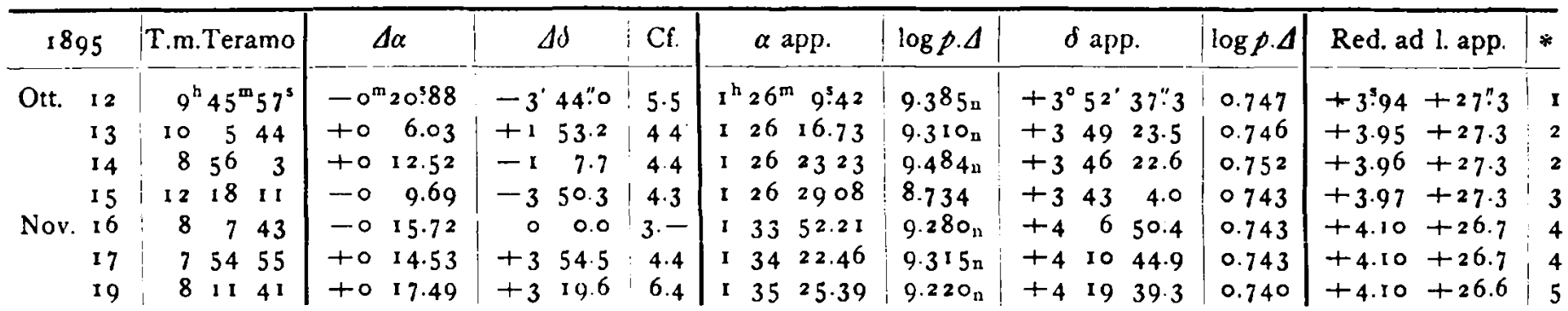

Stelle di confronto 1895.0 .

\begin{tabular}{|c|c|c|c|c|c|c|c|c|c|c|}
\hline * & Gr. & $\alpha$ & $\delta$ & Autorità & * & Gr. & $\alpha$ & & $\delta$ & Autorità \\
\hline 1 & 9.8 & $1^{\mathrm{b}} 26^{\mathrm{m}} 26^{\mathrm{s}} \cdot 3^{6}$ & $+3^{\circ} 55^{\prime} 54^{\prime \prime} .0$ & riferita $a * A$ & 4 & $9 \stackrel{\mathrm{m}}{6}$ & $1^{\mathrm{h}} 34^{\mathrm{m}} 3^{\mathrm{s}} 88_{3}$ & $+4^{\circ}$ & 6. 23.7 & riferita $a * C$ \\
\hline$A$ & 9.7 & I 2530.62 & +407.5 & AG. Albany 416 & C & 8.2 & 13628.89 & +4 & 830.5 & AG. Albany 480 \\
\hline 2 & I 11.0 & 1 26 & +3473.0 & riferita $a * B$ & 5 & 10 & I 35 & +41 & 1553.1 & riferita $a * D$ \\
\hline B & $\begin{array}{r}8.4 \\
11.0\end{array}$ & $\begin{array}{llr}1 & 27 & 4.75 \\
1 & 26 & 34.80\end{array}$ & $\begin{array}{l}+34236.4 \\
+34627.0\end{array}$ & $\begin{array}{l}\text { A. G. Albany } 423 \\
\text { riferita } a * B\end{array}$ & D) & 9.0 & I $34 \quad 39.99$ & +42 & 2327.5 & AG. Albany 467 \\
\hline
\end{tabular}

Ott. 12-15. Ingrandimento 250. La cometa fu sempre ben visibile, e solo il cattivo tempo pose fine alle osservazioni. - Nov. 16-19. Ingrandimento 200: puntate alquanto difficili. Cometa debolissima, diffusa circolarmente per 2' diametro. Nov. I6 si osservó la congiunzione della cometa colla stella di confronto, in Decl.

Teramo 1895 Dicembre.

V. Cerulli.

Beobachtung des Cometen $1895 \ldots$ (Brooks Nov. 21).

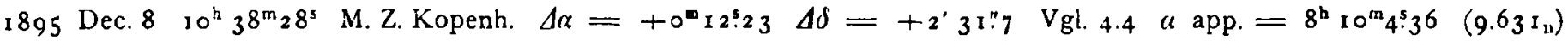
$\delta$ app. $=+44^{\circ} 46^{\prime} 42^{\prime \prime} 9(0.570)$

Vergleichstern $(c)-* a=+\mathrm{I}^{\mathrm{m}} 54^{\mathrm{s}} \cdot 84+\mathrm{I}^{\circ} \eta^{\prime \prime} \mathrm{I}$ Vgl. 3.2

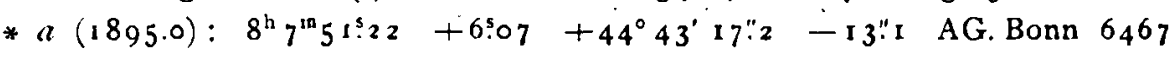

Comet schwach, war heute leichter zu pointiren. Da er dem Stern $(c)$ genügend nahe stand, wurden auch die $\Delta \alpha$ durch mikrometrische Messung, nicht durch Passagen erhalten.

Kopenhagen 1895 Dec. 9.

C. F. Pechiule.

Noner Planet 1895 CE. Planète Charlois 9 Décembre $9^{\mathrm{h}} 4^{\mathrm{m}} . \circ$ t. $\mathrm{m}$. Nice AR. $=66^{\circ} 7^{\prime}$ DP. $=7^{\circ} 3^{\prime}$. Mouv. diurne $-15^{\prime}+5^{\prime}$ Grandeur $1 \mathrm{I}^{\mathrm{m}}$.

Perrotin

Inhalt zu Nr. 3323. T. F. F. See. On the theoretical possibility of determining the distances of Star Clusters and of the Milky Way, and of investigating the Structure of the Heavens by actual measurement 16r. $-T$. F. F. See. Researches on the orbit of $\xi$ Ursae Majoris $=\Sigma$ 1523. 163. $-T: \mathcal{F}$. F. See. Researches on the orbit of $\gamma$ Coronae Australis. 169. - F. Plassmaann. Rauchkeilbeobachtungen von $\eta$ Aquilae. 17t. - F. Cohn. Beobachtungen des Cometen $1895 \ldots$ (Perrine Nov. 16). I73. -

V. Cerulli. Osservazioni della Cometa 1895 Il (Sivift). 175. - C. F. Pechüle. Beobachtung des Cometen 1895 ... (Brooks Nov. 21). 175. - Perrotin. Neuer Planet 1895 CE. 175. 